University of Wollongong

Research Online

Faculty of Engineering - Papers (Archive)

Faculty of Engineering and Information

Sciences

$1-1-2009$

\title{
Packaging effects on RadFET sensors for high energy physics experiments
}

\author{
Julien Mekki \\ CERN \\ Laurent Dusseau \\ University Montpellier II, France \\ Maurice Glaser \\ CERN \\ Susanna Guatelli \\ ANSTO, susanna@uow.edu.au \\ Michael Moll \\ CERN
}

See next page for additional authors

Follow this and additional works at: https://ro.uow.edu.au/engpapers

Part of the Engineering Commons

https://ro.uow.edu.au/engpapers/2560

\section{Recommended Citation}

Mekki, Julien; Dusseau, Laurent; Glaser, Maurice; Guatelli, Susanna; Moll, Michael; Pia, Maria Grazia; and Ravotti, Federico: Packaging effects on RadFET sensors for high energy physics experiments 2009, 2061-2069.

https://ro.uow.edu.au/engpapers/2560

Research Online is the open access institutional repository for the University of Wollongong. For further information contact the UOW Library: research-pubs@uow.edu.au 


\section{Authors}

Julien Mekki, Laurent Dusseau, Maurice Glaser, Susanna Guatelli, Michael Moll, Maria Grazia Pia, and Federico Ravotti 


\title{
Packaging Effects on RadFET Sensors for High Energy Physics Experiments
}

\author{
Julien Mekki, Laurent Dusseau, Senior Member, IEEE, Maurice Glaser, Susanna Guatelli, Member, IEEE,
} Michael Moll, Maria Grazia Pia, and Federico Ravotti, Member, IEEE

\begin{abstract}
RadFETs in customized chip carrier packages are installed in the LHC Experiments as radiation monitors. The package influence on the dose measurement in the complex LHC radiation environment is evaluated using Geant4 simulations and experimental data.
\end{abstract}

Index Terms-Dose enhancement, Geant4, Monte Carlo, particle beams, RadFET, radiation monitoring.

\section{INTRODUCTION}

A $\mathrm{T}$ the Large Hadron Collider (LHC), the complex radiation environment will be composed of charged hadrons, neutrons, electrons and photons [1], [2]. All detector and electronic components present in this particle field are potentially affected by radiation damage [3]. For this reason, the radiation fields in the LHC have to be precisely characterized and monitored. In the framework of the RADMON project several radiation sensors have been evaluated for this purpose [4], [5]. Finally, integrated sensor boards including RadFETs (Radiation-sensing Field-Effect Transistors) for Total Ionizing Dose (TID) measurements, $\mathrm{p}-\mathrm{i}-\mathrm{n}$ diodes to record the equivalent particle fluence and temperature sensors have been developed [6], produced and installed in the LHC experiments ALICE, ATLAS [7], LHCb [8] and TOTEM [9].

RadFETs are p-channel MOSFET Transistors optimized for dose measurement [3]. The radiation sensitivity of these FETs is achieved by a thicker gate oxide compared to conventional MOSFETs. Radiation induced charge trapping at the $\mathrm{Si} / \mathrm{SiO} 2$ interface causes a variation of the threshold voltage (Vth), which is measured at a constant source-drain current. Vth is then a measure for the dose deposited in the gate oxide. Even assuming that the particle spectrum in the experiments is sufficiently well predicted by Monte Carlo simulations, the question remains how much dose different particles with different energies deposit in the RadFETs. As not all particles and particle energies can be evaluated experimentally, simulations are needed for converting the particle fluence to measured dose for all particle types and particle energies.

Manuscript received October 10, 2008; revised January 16, 2009. Current version published August 12, 2009.

J. Mekki is with CERN, CH1211 Geneva 23, Switzerland, and also with IES, Montpellier, France (e-mail: Julien.Mekki@cern.ch).

M. Glaser, M. Moll, and F. Ravotti are with the CERN, CH1211 Geneva 23, Switzerland

S. Guatelli is with the ANSTO, Menai NSW 2234, Australia.

M. G. Pia is with the INFN Sezione di Genova, Genova 16146, Italy.

L. Dusseau is with the IES-UMR UMII-CNRS 5214, University Montpellier II, F-34095, Montpellier Cedex 5, France.

Color versions of one or more of the figures in this paper are available online at http://ieeexplore.ieee.org.

Digital Object Identifier 10.1109/TNS.2009.2014376
A further complication in interpretation of the measured dose values is arising from the packaging of the RadFETs. The package can alter the measured dose by various mechanisms (absorption, attenuation, creation of secondary particles, etc.). A clear understanding of the influence of the package in the particular radiation field of interest is therefore crucial to optimize the package or at least understand its impact on the measurement. Only few experimental works have been reported on this topic. For example, the increase of the dose measured with RadFETs exposed to fast neutrons and covered with Polyethylene slabs of different thicknesses was investigated in [10]. RadFETs exposed to a Co-60 source and to monoenergetic low energy protons, covered or not covered by a lid were investigated in [11]. For MOSFET devices exposed to low-energy photons an increase of the measured TID due to the packaging has been reported in [12], [13].

In this work we study the expected response of RadFETs in the complex radiation fields of the LHC experiments using the Geant4 toolkit [14], [15]. A Geant4 application was developed describing the geometry and material of the RadFET sensors and the surrounding package in great detail. An assessment on the validity of the physics models used for the application was performed to evaluate the reliability of the performed simulations for various particles and particle energies being present in High Energy Physics experiments radiation fields. Finally, the application was used to optimize the package of the RadFETs. To our best knowledge a similar approach for the packaging optimization of a FET based dosimeter has been performed only once. However, the work described in [11] is aiming for space applications and is limited to low energy protons, while our study spans over a multitude of particles and a wide particle energy range up to $100 \mathrm{GeV}$.

In the following section we describe the RadFETs and the package. Furthermore, experimental data obtained with fast neutrons and $254 \mathrm{MeV}$ protons [16] using the RadFETs and the type of packaging under consideration in this work are presented. In Section III the Geant 4 application and its validity is described and discussed. In Section IV, simulations describing package impact on the dose measurement for various particles are presented and finally, in Section V, the influence of the packaging on the RadFETs response in typical LHC experiment radiation fields is presented and discussed.

\section{RADFET PACKAGE AND EXPERIMENTAL RESULTS}

\section{A. Chip Carrier for Radfets at the LHC Experiments}

Design studies were performed by the CERN Physics Department in order to find the best suitable package for RadFET dosimeters to be used in the LHC experiments [4]. The selected 


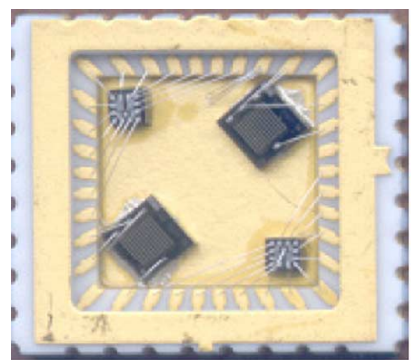

(a)

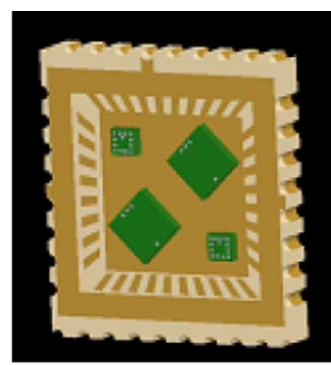

(b)
Fig. 1. Chip carrier used for the LHC experiments. Four RadFETs of 2 different types are mounted and wire-bonded: (a) Photo; (b) Front view of the chip carrier in the Geant 4 model.

package is an Alumina $\left(\mathrm{Al}_{2} \mathrm{O}_{3}\right)$ chip carrier with a size of $1 \times$ $1 \mathrm{~cm}^{2}$ and a thickness of $1.3 \mathrm{~mm}$. It allows mounting of up to 4 RadFETs inside the carrier as illustrated in Fig. 1(a). Two types of RadFETs are used: The REM TOT-501C supplied by the REM Oxford Ltd, UK and the LAAS 1600 manufactured by the Laboratory of Analysis and Architecture of System of CNRS in France. They have an oxide thickness of $250 \mathrm{~nm}$ and $1600 \mathrm{~nm}$ and a size of $0.95 \times 0.95 \mathrm{~mm}^{2}$ and $1.9 \times 1.9 \mathrm{~mm}^{2}$, respectively. The RadFETs are wire-bonded and connected via an $\mathrm{Au} / \mathrm{W}$ metallization (backplane and bond pads) of about $3 \mu \mathrm{m}$ thickness. The number of sensors in the carrier supplied to the LHC experiments varies from 1 to 4 according to the specific needs of the experiments [16]. For this reason, the connection layout for all pins of the carrier and the dosimeters has been standardized [4].

Having fixed the type of carrier and its connection scheme for the development of the read-out electronics and the carrier mounting in the experiments, the only freedom remaining in the design of the package is the chip carrier cover. It will protect the RadFETs against dust and mechanical damage. However, like the chip carrier itself, it has also an impact on the TID measurement which is evaluated in this work.

\section{B. Experiments}

Experimental studies were carried out at the High Energy Proton Facility (PIF) of the Paul Scherrer Institute (PSI) in Villigen, Switzerland [17] and at the Reactor Research Center of the Joseph Stephan Institute (JSI) in Ljubljana, Slovenia. At JSI, the neutron irradiations were performed in a reactor of type TRIGA [18]. The neutron spectrum available in this facility ranges from thermal to fast neutrons of about $1 \mathrm{MeV}$. The RadFET carriers were irradiated by placing them into the reactor core through an irradiation tube that occupies a fuel rod position. At PIF the carriers were irradiated perpendicularly to the beam-axis with $254 \mathrm{MeV}$ protons at an average flux of about $1.0 \times 10^{8}$ proton $/ \mathrm{sec} / \mathrm{cm}^{2}$. In order to evaluate the influence of the chip carrier cover on the dose measurement, irradiations at both facilities were performed by increasing the fluence in steps and by covering the chip carrier with different thicknesses of different materials during the various irradiation steps.

The results are presented in Fig. 2 where the materials and thicknesses of the covers are indicated for each irradiation step. Results showed that for $254 \mathrm{MeV}$ protons, the different covers had no effect on the TID measured. The experiment at the nuclear reactor revealed that most of the used covers are trans-

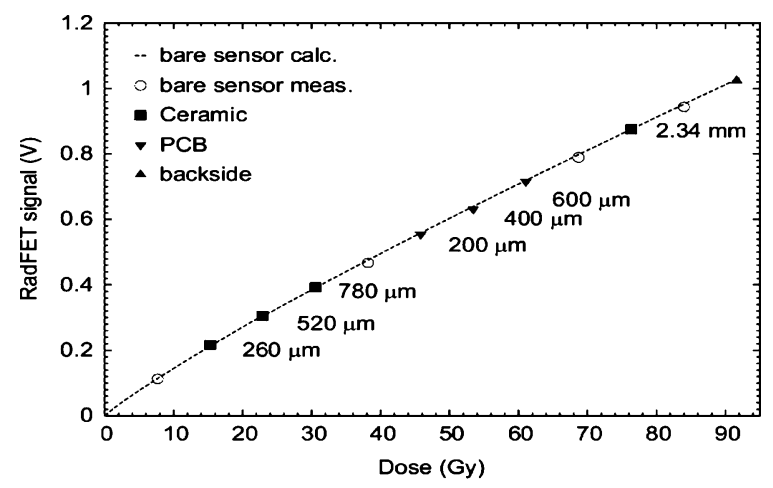

(a)

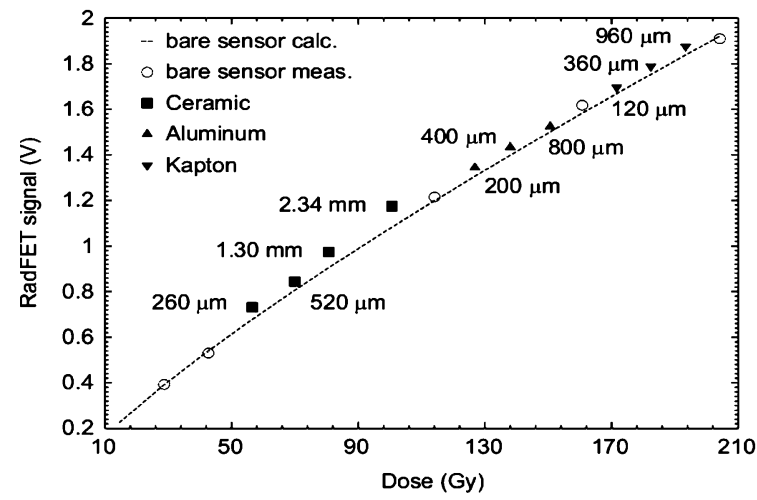

(b)

Fig. 2. Experimental results of the REM-TOT-501C obtained (a) at PSI with $254 \mathrm{MeV}$ protons. (b) Experimental results obtained at JSI during neutron irradiation. Error bars are smaller than the symbol size.

parent to neutrons. Only for Alumina covers exceeding a thickness of $1 \mathrm{~mm}$ a slight increase of the TID was observed. A more detailed description of the experiments is given in [16], [19]. A brief comparison with simulation results is described in Section IV-A.

\section{GEANT4 APPLICATION}

A simulation application was developed specifically for this study, exploiting the Geant4 toolkit. It models the detector with various packaging options and the radiation it is exposed to, it configures the selection of Geant 4 physics to be activated, and it scores the energy deposited in the sensor.

The detector model in the simulation is based on a detailed description of the geometry and materials of the sensors and the chip carrier. Fig. 1(b) shows the model of the detector geometry. The four RadFETs are represented by four dies placed on an Alumina chip carrier with a size of $1 \times 1 \mathrm{~cm}^{2}$ and a total thickness of $1.3 \mathrm{~mm}$. The die sizes and oxide thicknesses are given in Table I. In a first set of simulations, discussed in Section IV, a preliminary model was adopted: the full dies, consisting of epoxy-glass, are taken as sensitive volume, where the energy deposit is scored. The simulations presented in Section $\mathrm{V}$ were performed with a more detailed detector model: the sensitive volume was reduced to represent only the thin oxide layer $\left(\mathrm{SiO}_{2}\right)$ of the RadFETs. The two geometry models were verified to produce consistent results, as discussed in Section V. The radiation environment is modeled as a monochromatic beam orthogonally incident on the chip carrier. Complex radiation environments were studied as a superposition of results 
TABLE I

GEOMETRY OF THE Four DIES REPRESENTING THE RADFET SENSORS IN THE GEANT4 APPLICATION.

\begin{tabular}{ccccc}
\hline \hline Device & $\begin{array}{c}\mathrm{x} \\
(\mathrm{mm})\end{array}$ & $\begin{array}{c}\mathrm{y} \\
(\mathrm{mm})\end{array}$ & $\begin{array}{c}\mathrm{z} \\
(\mathrm{mm})\end{array}$ & $\begin{array}{c}\text { Oxide } \\
(\mathrm{nm})\end{array}$ \\
\hline REM-TOT-501C & 0.95 & 0.95 & 0.405 & 250 \\
\hline LAAS-1600 & 1.9 & 1.9 & 0.405 & 1600 \\
\hline \hline
\end{tabular}

from different simulations with monochromatic beams, each one characterized by a given energy and particle type. The particle beam size was $1.2 \times 1.2 \mathrm{~cm}^{2}$, which is slightly bigger than the $1 \times 1 \mathrm{~cm}^{2}$ chip carrier; the larger transversal beam section was meant to exclude border effects.

A Geant4-based simulation requires that all the particle species involved are instantiated, and their interaction processes are specified [14]; for many physics processes Geant4 offers the option to choose among different models. Both electromagnetic and hadronic physics processes were activated in the Geant 4 application, to describe the interactions of the incident particle, and of all the secondary particles produced.

The electromagnetic processes activated in the simulation encompassed ionization and multiple scattering for all charged particles, Bremsstrahlung for electrons, photoelectric effect, Compton and Rayleigh scattering, and pair production for photons. The Low Energy [20], [21] model based [22] on the ICRU49 [23] parameterizations was selected for hadron ionization. The models for electrons and photons in the Low Energy or in the Standard [24] Electromagnetic packages were alternatively activated in the simulation; specifically, among the low energy ones [25], those based on the EEDL [26] and EPDL97 [27] data libraries, The Rayleigh scattering was activated only in connection with the usage of the Low Energy Electromagnetic package, since this process is not implemented in the Standard Electromagnetic package.

The selection of the models used in the simulation is supported by documented validation.

The above mentioned electromagnetic models have been compared [28] to the authoritative NIST Physical Reference Data [29]; both the Standard and Low Energy ones were demonstrated to be in agreement against the reference. Further validation results of Low Energy electromagnetic models against precision measurements of energy deposited by low energy incident electrons are documented in [30]. Various models of proton ionization are available in the Geant4 Low Energy package, respectively based on parameterizations in [23], [31]-[33]; they exhibit different behavior [34] in the low energy domain (below $2 \mathrm{MeV}$ ). Their differences amount to a few percent at most with respect to the NIST Reference Data; the one chosen in the application is equivalent to the NIST Reference data at the $90 \%$ confidence level. Such differences are not critical to the study presented in this paper.

Muon interactions are simulated through the processes in the Geant4 Standard muon package [35].

The multiple scattering process [36] was activated according to its default configuration. Recent studies [30] have documented its contribution to the accuracy of dosimetry simulations.

Hadronic elastic and inelastic processes were activated in the simulation; they were articulated through different models according to the energy range and particle type. The fission and capture processes were active for neutrons. The design of Geant 4 hadronic physics package allows the user to select different cross section and final state models associated with a given process.

Hadronic elastic scattering is treated according to the Low Energy Parameterized model, which is based on original parameterizations from GHEISHA [37].

Default inelastic cross sections were used in the simulation.

Several models were selected to generate hadronic inelastic final states in different energy ranges; their activation ranges partly overlapped to ensure a smooth transition between different simulation regimes.

The binary cascade model [38] was active in the lower energy range (up to $10 \mathrm{GeV}$ ) for protons, neutrons and pions; it encompasses models for intranuclear transport, pre-equilibrium phase and nuclear de-excitation. At intermediate energies (8-25 GeV) the Parameterized models, originally derived from GHEISHA [37], were activated. The highest energy range ( $20 \mathrm{GeV}-100 \mathrm{TeV}$ ) was covered by the Quark Gluon String model [39].

The inelastic hadronic scattering of deuteron, triton and $\alpha$ particles was modeled with the Low Energy Parameterized model up to $25 \mathrm{GeV}$ and the Binary Light Ion model up to $10 \mathrm{GeV} /$ nucleon; Tripathi [40]-[43] and Shen [44] cross sections were selected. The Binary Ion Model was active for ions.

The validation of the hadronic interaction models used in the simulation is documented in [45]-[48]; it is worth remarking, however, that while the validity of the Geant 4 electromagnetic models used is well established, the validation of the hadronic ones is still in progress, due to the complexity of the physics domain.

A Geant4-based simulation requires that the user defines a threshold for the production of secondary particles from processes affected by infrared divergence [14]; the secondary production threshold is expressed in terms of range. The secondary production threshold affects the precision of the energy deposit distribution; its setting should be consistent with the desired precision and the geometrical characteristics of the simulated experimental set-up. The production threshold of secondary particles was fixed to $1 \mu \mathrm{m}$ for the simulations in Section IV and $0.2 \mu \mathrm{m}$ for simulations in Section V; in both cases these settings were significantly smaller than the thickness of the sensitive volume associated with the detector model, thus ensuring the accuracy of the spatial distribution of the energy deposit in the simulation.

The energy deposited in the four dies (respectively oxide layers for simulations in Section V) is scored as a result of the simulation; it accounts for the interactions of primary and secondary particles with the target volume. The average energy deposited per incident primary particle per unit area is calculated by scaling this value by the number of primary particles hitting the chip carrier and its area.

The simulations were performed with Geant 4 version 8.0. 


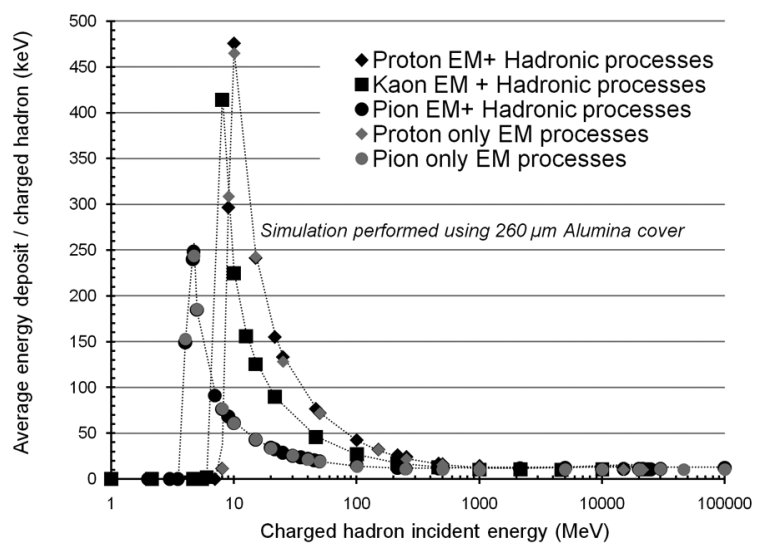

Fig. 3. Average energy deposited in the four RadFET sensors per incident particle for charged hadrons resulting from electromagnetic (EM) interactions only and by both electromagnetic and hadronic ones. Simulations were performed with an Alumina cover thickness of $260 \mu \mathrm{m}$.

\section{RAdFet Packaging EFFects Evaluated USING GEANT4 SimULATIONS}

The packaging effect was investigated by studying the energy deposited by primary and secondary particles in the sensitive volume using different thicknesses of the chip carrier lids: $260 \mu \mathrm{m}, 780 \mu \mathrm{m}, 2340 \mu \mathrm{m}$ and without any lid. One of the issues under study is the contribution of secondary particles to possible dose enhancements.

The following sections report the results of the analysis for various kinds of primary particles; when the error bars are not explicitly reported in the figures, the statistical uncertainties of the simulation results are smaller than the size of the symbols.

\section{A. Results for Charged Hadron Simulations}

The energy deposit resulting from incident protons, pions and kaons was investigated in the simulations.

The contribution to the energy deposit deriving from electromagnetic and hadronic interactions was evaluated by comparing simulations where both types of processes were activated, to those where electromagnetic interactions only were active in the Geant4-based application. Hadronic interactions can modify the energy deposited in the sensitive volume through different effects: elastic scattering affects the particle direction, therefore the path it traverses in the detector and the resulting energy loss; inelastic interactions affect the energy loss through the possible suppression of the primary particle and the creation of secondary ones, whose energy deposit may differ significantly from the one due to the electromagnetic interactions of the primary particle only [34]. The results are shown in Fig. 3 for protons, pions and kaons; they concern an energy range similar to what is expected in realistic operation conditions at LHC.

A statistical analysis was performed to evaluate the degree of compatibility of the energy deposit distributions deriving from simulations where only electromagnetic interactions were active with respect to simulations accounting for both electromagnetic and hadronic interactions. The Kolmogorov-Smirnov [49], [50] goodness-of-fit test was applied to compare the two distributions respectively for protons and pions shown in Fig. 3; the null hypothesis stated their compatibility. A confidence level of 0.1 was

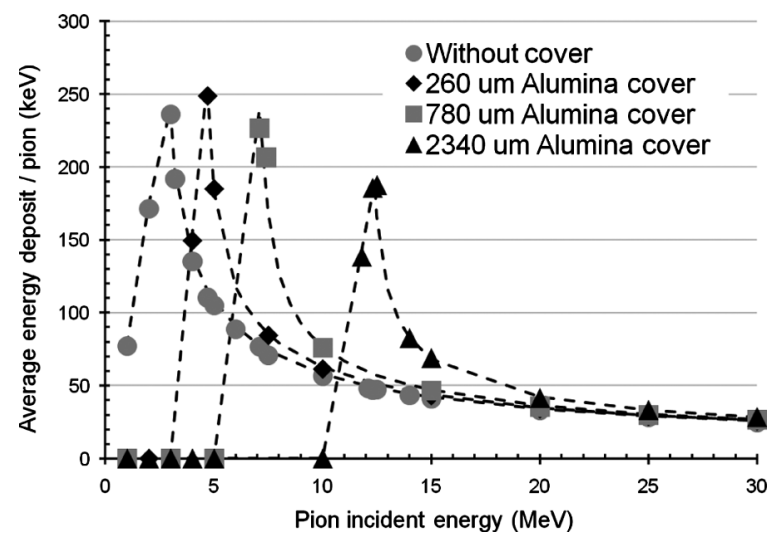

Fig. 4. Average energy deposited in the four RadFET sensors per incident particle for pions. Simulations were performed with or without Alumina cover of the thickness indicated in the figure.

set to define the critical region determining the rejection of the null hypothesis; the p-value resulting from the test was 0.589 and 0.937 respectively for protons and pions, thus failing to reject the null hypothesis in both cases. Similar behaviour was verified with kaons.

This result demonstrates that the energy deposited by charged hadrons in the RadFET sensors is mostly due to electromagnetic interactions, while hadronic processes contribute to a lesser degree. Therefore the accuracy of the results presented in this study is dominated by the accuracy of the Geant 4 electromagnetic processes used in the simulation, which is discussed in the previous section: this gives confidence into the predictive capabilities of the simulation. This result also shows that no significant dose contribution from secondary particles created by nuclear interactions of charged hadrons in the cover is expected.

For the same kinetic energy protons depose more energy than kaons and pions due to their higher mass, leading to a higher ionization density in the traversed material. Protons are therefore absorbed in the cover up to higher energies. Fig. 4 presents pion simulations performed with the Geant 4 application indicating how the energy deposit in the RadFETs depends on the cover thickness and the pion energy. The amount of material in the lid modulates the fraction of lower energy primary particles which are absorbed in the cover and the creation of secondary particles, which in turn contribute to the energy deposit observed in the sensitive volume. This effect is illustrated in Fig. 5, which compares the average energy deposit with a $260 \mu \mathrm{m}$ Alumina lid and without any cover as a function of pion energy; the energy bins correspond to a realistic radiation spectrum expected in the LHC experiments [51]. The dose enhancement at higher energies due to the cover is of the order of $10 \%$.

Finally it has to be mentioned that simulations have been performed simulating the $254 \mathrm{MeV}$ proton experiment described in the previous section. The simulations predict, as observed in the experiment, that no dose enhancement for the used cover materials and thicknesses is expected.

\section{B. Results for Electrons}

A significant fraction of the electrons up to several $\mathrm{MeV}$ are absorbed in the Alumina cover. As a consequence a smaller frac- 


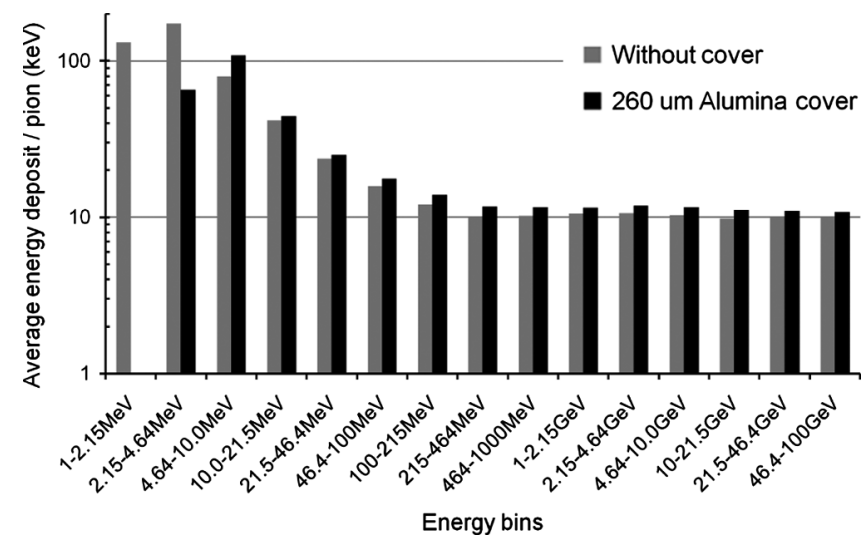

Fig. 5. Average energy deposited in the four RadFET sensors per incident particle for pions. Simulations were performed with or without Alumina cover thickness of $260 \mu \mathrm{m}$.

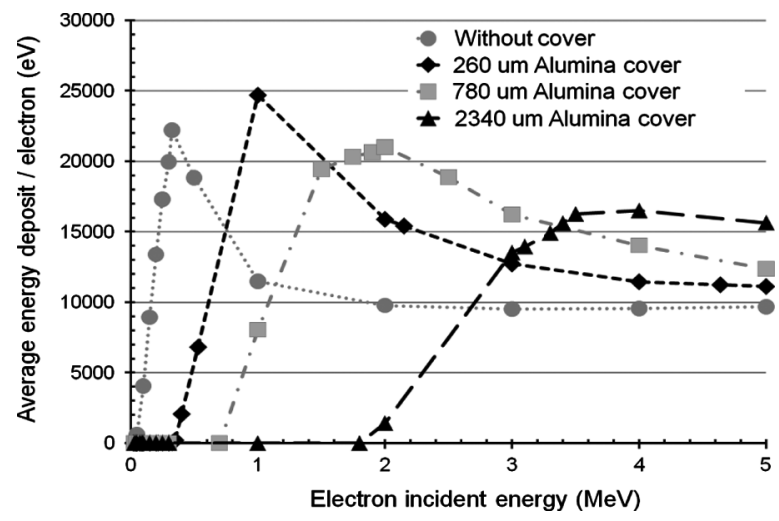

Fig. 6. Average energy deposit per incident electron in the four RadFET sensors covered or not covered with Alumina slabs of different thicknesses.

tion of the incident particles contribute to the energy deposited in the sensor than for charged hadrons for which the cover reduces the energy of the particles but not their flux, unless they are completely stopped in the cover.

Fig. 6 shows simulations for the average energy deposition in the RadFETs by electrons of up to $5 \mathrm{MeV}$ kinetic energy, assuming different Alumina thicknesses used to cover the RadFETs. The simulations visualize that for RadFETs covered with a $2340 \mu \mathrm{m}, 780 \mu \mathrm{m}$ or $260 \mu \mathrm{m}$ Alumina slabs, the energy cut-off appears around incident electron energies of $2 \mathrm{MeV}$, $700 \mathrm{keV}$ or $300 \mathrm{keV}$, respectively. This is consistent with the Number Transmission Coefficient (NTC) [52].

\section{Results for Photons}

The total mass attenuation coefficient for photons in Alumina is tabulated at NIST-XCOM [53]. It is composed of contributions arising from the photoelectric effect, the Compton effect and pair production which are reflected in the Geant 4 simulations presented in Fig. 7. The average energy deposit in the RadFETs for photons of up to $140 \mathrm{keV}$ is shown in Fig. 7(a). Due to the strong photoelectric effect the photons are quickly absorbed even in thin layers of Alumina resulting in a strong difference in the deposited dose between covered and non covered RadFETs for photons of up to $40 \mathrm{keV}$. A peak in the average energy deposit is observed at about 10 to $20 \mathrm{keV}$ when the photons are

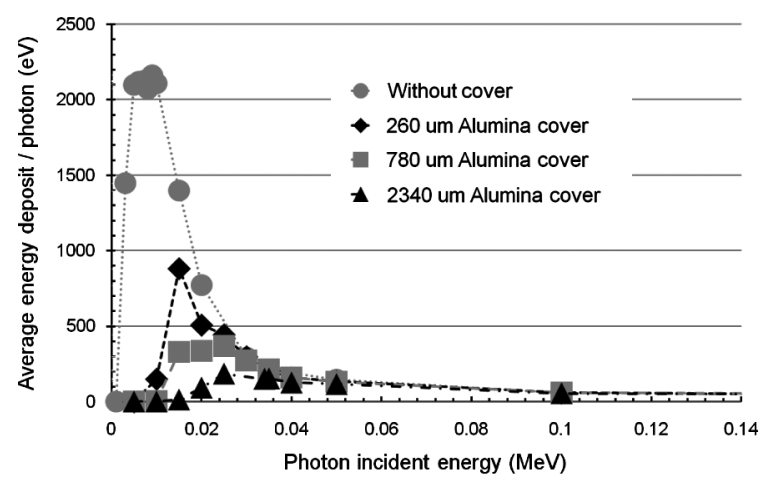

(a)

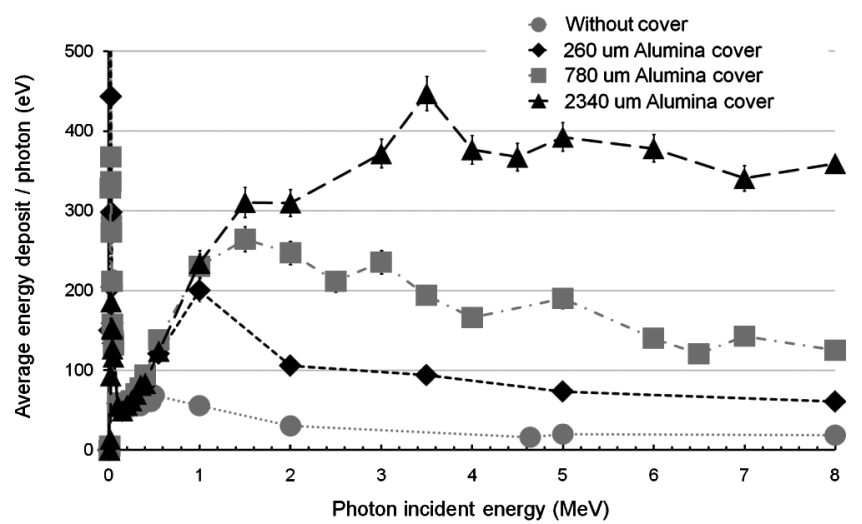

(b)

Fig. 7. Simulation results for average energy deposit per event in the sensitive volume using three different Alumina thicknesses. (a) Zoom to the average energy deposit at low energy photons. (b) Over a photon energy range from $5 \mathrm{keV}$ to $8 \mathrm{MeV}$.

almost completely absorbed, while for higher energies the photons start to traverse the cover and the RadFETs.

At photon energies higher than $40 \mathrm{keV}$ the Compton effect starts to dominate. The second peak in the average energy deposit of the RadFETs shown in Fig. 7(b) arises from Compton electrons created outside the sensitive volume. The peak reflects the rising and falling of the Compton effect mass attenuation coefficient with photon energy as well as the decrease of energy deposition for electrons with rising energy.

For energies above about $5 \mathrm{MeV}$, also secondary electrons and positrons created by the pair effect contribute to the energy deposit. Finally, for even higher energies photonuclear absorption processes occur [54]. Although their total cross sections are small, they could contribute to the dose enhancement in the high energy range, as the resulting protons or alpha particles deposit a significant amount of energy in the sensitive RadFET layer. The physics configuration in the simulation application accounts for this kind of processes through the models mentioned in Section III. Geant 4 includes physics models [55], [56] specifically meant to handle photonuclear processes in detail; however, such a study goes beyond the scope of the present paper and would be addressed in a dedicated project. In the present study the production of $\alpha$ particles contributing significantly to the dose released in the RadFETs appears with a frequency of the order of one event per million primary photons. In general the dose enhancement is more important for 


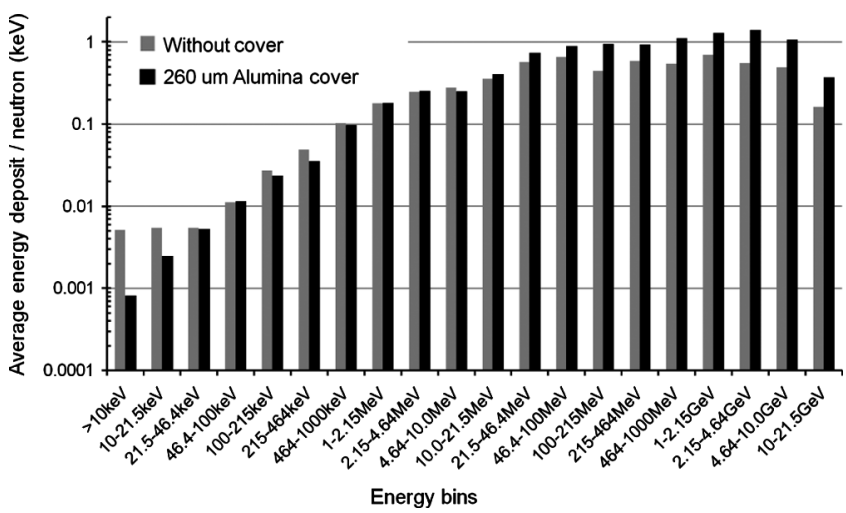

Fig. 8. Average energy deposited in the four RadFET sensors per incident particle for neutrons. Simulations were performed with or without Alumina cover thickness of $260 \mu \mathrm{m}$.

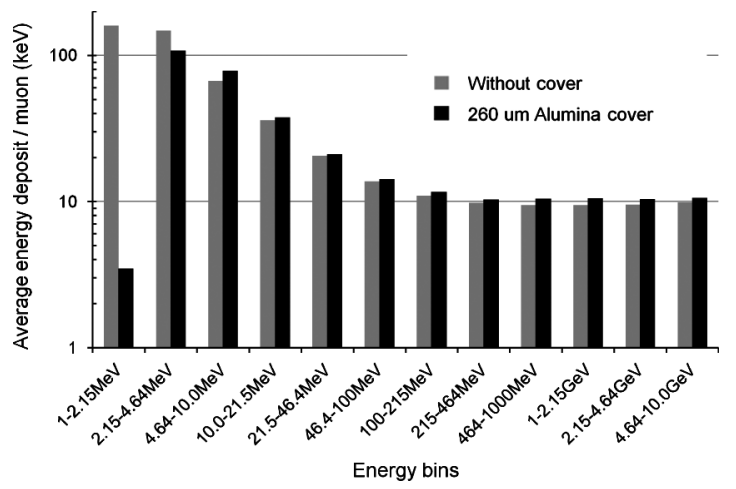

Fig. 9. Average energy deposited in the four RadFET sensors per incident particle for muons. Simulations were performed with or without Alumina cover thickness of $260 \mu \mathrm{m}$.

sensors covered by thick Alumina lids due to the more significant number of secondary particles generated within the thicker package.

\section{Results for Neutrons}

A preliminary evaluation has been performed concerning incident neutrons. Their effect is illustrated in Fig. 8, which compares the average energy deposit with a $260 \mu \mathrm{m}$ Alumina lid and without any cover as a function of neutron energy corresponding to a realistic radiation spectrum in the LHC environment: a dose enhancement is observed at higher energies. A more detailed appraisal of the effect of neutrons would require dedicated simulations with the specialized "High Precision" models for neutron interactions available in the Geant4 Toolkit; it should be noted, however, that, according to the preliminary results, in the realistic operation environment described in the following section neutrons contribute by approximately $10 \%$ to the energy deposit in the RadFETs, while they represent a major component in the radiation spectrum.

\section{E. Results for Muons}

The cover material has the effect of stopping low energy muons, while some effect of dose enhancement is observed at higher energies, as documented in Fig. 9. This simulation derives from a primary muon spectrum as expected in realistic operation conditions in the LHC experiments.

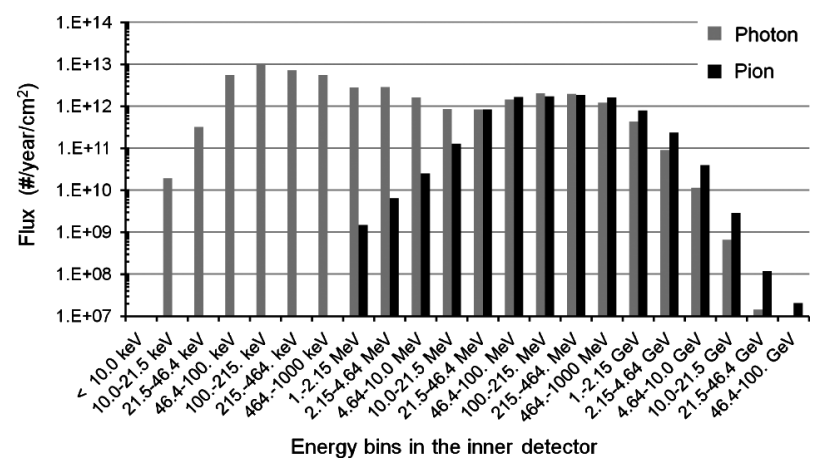

Fig. 10. Photon and pion spectra for ATLAS inner detector (SCTB1).

\section{RADFET SENSORS IN THE ATLAS DETECTOR}

The RadFET sensors described in this work will provide online information about the TID at various locations inside the LHC experiments.

The radiation fields in two specific locations of ATLAS are taken here as an example to understand the influence of the package on the measured dose in typical LHC experiment spectra. The first location, labeled SCTB1, is situated inside the inner detector and the second one, labeled LAR Barrel, is located in the Liquid Argon Calorimeter.

The spectra corresponding to these locations [51] give the number of expected particles per year and $\mathrm{cm}^{2}$ per energy bin assuming "annual predictions" for $10^{7}$ seconds at high luminosity $\left(10^{34} \mathrm{~cm}^{-2} \mathrm{~s}^{-1}\right)$; they derive from Monte Carlo simulations based on FLUKA [57], [58] and GCalor [59]. Statistical uncertainties for the total flux values of each particle type are less than 3\% for the SCTB1 location while they are lower than $7 \%$ for the LAR Barrel position. As an example, the photon and pion spectra for the SCTB1 location are illustrated in Fig. 10.

To estimate the contribution of each particle type to the energy deposited in the RadFET and the packaging effects, a Geant 4 simulation for each particle type (protons, photons, electrons, pions, neutrons, muons) and energy bin has been performed. The calculations were performed with an Alumina lid of $260 \mu \mathrm{m}$ thickness, which has tentatively been defined as the cover to be used in the LHC experiments.

A more detailed geometrical model of the detector was implemented for these simulations. In the simulations presented in the previous chapters the total volume of the RadFET dies was taken as sensitive volume. This allowed for the relative comparison of the measured energy deposition using different packaging solutions. However, only the charge deposited in the oxide layer of the FET on the RadFET die actually contributes to the RadFET signal; this very thin oxide layer is located close to the surface of the RadFET die. For the simulations presented in this section therefore a RadFET geometry composed of 3 different layers was used: a $1600 \mathrm{~nm} \mathrm{SiO}$ layer, representing the sensitive volume, sandwiched between a $0.5 \mu \mathrm{m}$ Chrome layer on the top and a $400 \mu \mathrm{m}$ Silicon layer below. This detailed geometrical model allows one to understand possible thickness effects of the sensitive volume on the dose measurement, and to improve the precision of the absolute dose values predicted by the simulations. 


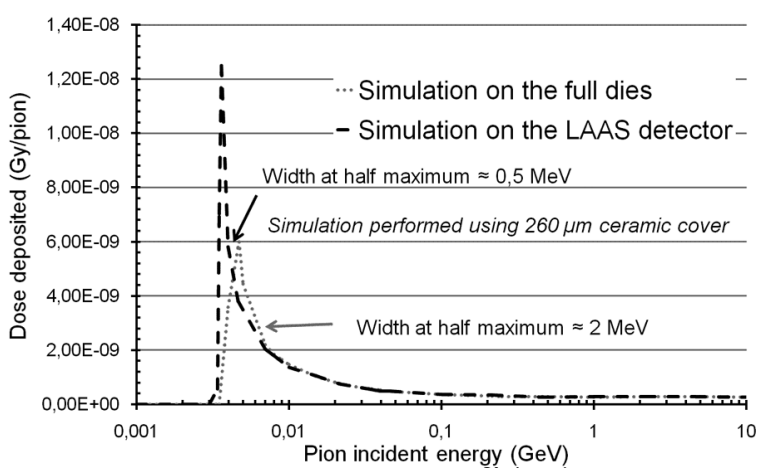

Fig. 11. Geant 4 simulations of the average deposited dose per pion. The results in both detector geometries used in this work are compared.

The energy deposit scored in the detailed geometrical model is largely equivalent to the one where the full RadFETs die represents the sensitive volume, with the exception of the low energy range, in which the particles are either completely stopped or are still close to the energy region of the Bragg peak. For higher energies, when the $\mathrm{dE} / \mathrm{dx}$ can be regarded as constant over the full thickness of the sensitive volume, no difference is observed any more. An example is illustrated in Fig. 11, which gives a comparison between the average deposited dose in unit of Gy/pion for the two geometries. The different behaviour of the two geometries would become relevant only in the case when a significant part of the pion spectrum in the operation environment would fall into the low energy range below $10 \mathrm{MeV}$ identified in Fig. 11; this is not the case expected in the LHC radiation field. No significant difference appears to be associated to the different materials (epoxy-glass and silicon oxide) considered in the two simulation models; the only visible difference at low energy is due to the different thicknesses in the two geometries. Therefore the results discussed in the previous chapter, deriving from a simplified geometrical model, hold their validity also when a more detailed geometry is applied.

The results for the SCTB1 position are given in Table II; both this table and the following Table III do not report errors for better readability. The first column indicates for each particle type its fraction in the spectrum. In the second and third column the total annual dose is given for each particle using RadFET with and without cover, as well as the percent contribution of the overall annual dose. The last column reports the dose enhancement.

Although neutrons represent a quarter of all particles, their total energy deposited is less than $1 \%$ of the total, due to their low interaction probability with the sensor material. A significant dose enhancement of about $200 \%$ is observed for photons when a cover is placed on the RadFET, as expected from the discussion in Section IV-C. However, as the overall deposited dose by photons is only about $1 \%$, this dose enhancement does not play a role at this detector position.

The main contribution to the deposited energy is delivered by pions (about $45 \%$ ), while they represent only about $10 \%$ of all particles. The total dose enhancement expected in the SCTB1 spectrum by placing a cover on the RadFET is $(12.1 \pm 1) \%$. The annual TID expected to be measured is $(6.47 \pm 0.04) \mathrm{kGy} / \mathrm{year}$ without cover and $(7.25 \pm 0.05) \mathrm{kGy} /$ year with cover, indicating
TABLE II

SUMMARY OF SIMULATIONS MADE FOR THE INNER DETECTOR.

\begin{tabular}{ccccc}
\hline \hline & $\begin{array}{c}\text { \% of the } \\
\text { total } \\
\text { number of } \\
\text { particles }\end{array}$ & $\begin{array}{c}\text { Annual dose without } \\
\text { cover in units of } \\
\text { kGy/year } \\
\text { (contribution \%) }\end{array}$ & $\begin{array}{c}\text { Annual dose with } \\
\text { cover in units of } \\
\text { kGy/year } \\
\text { (contribution \%) }\end{array}$ & $\begin{array}{c}\text { Dose } \\
\text { enhancement } \\
(\%)\end{array}$ \\
\hline Protons & 1.2 & $1.73(26.7)$ & $1.65(22.8)$ & -4.3 \\
\hline Photons & 54.9 & $2.46 \times 10^{-2}(0.4)$ & $8.29 \times 10^{-2}(1.1)$ & 237.1 \\
\hline Electrons & 5.9 & $1.13(17.4)$ & $1.34(18.5)$ & 19.4 \\
\hline Pions & 10.8 & $2.89(44.7)$ & $3.39(46.7)$ & 17.2 \\
\hline Neutrons & 25.2 & $3.17 \times 10^{-2}(0.5)$ & $3.72 \times 10^{-2}(0.5)$ & 17.2 \\
\hline Muons & 1.9 & $6.66 \times 10^{-1}(10.3)$ & $7.42 \times 10^{-1}(10.2)$ & 11.5 \\
\hline Total & $\mathbf{1 0 0}$ & $\mathbf{6 . 4 7}$ & 7.25 & 12.1 \\
\hline
\end{tabular}

TABLE III

SUMMARY OF SIMULATIONS MADE FOR THE LIQUID ARGON CALORIMETER.

\begin{tabular}{ccccc}
\hline & $\begin{array}{c}\text { \% of the } \\
\text { total } \\
\text { number of } \\
\text { particles }\end{array}$ & $\begin{array}{c}\text { Annual dose } \\
\text { without cover in } \\
\text { units of Gy/year } \\
\text { (contribution \%) }\end{array}$ & $\begin{array}{c}\text { Annual dose } \\
\text { with cover in } \\
\text { units of Gy/year } \\
\text { (contribution \%) }\end{array}$ & $\begin{array}{c}\text { Dose } \\
\text { enhancement } \\
(\%)\end{array}$ \\
\hline Protons & 0.08 & $1.96(39.5)$ & $1.81(29.6)$ & -7.4 \\
\hline Photons & 42.3 & $2.47 \times 10^{-1}(5)$ & $8.62 \times 10^{-1}(14)$ & 248.9 \\
\hline Electrons & 0.6 & $1.65(33.3)$ & $2.16(35.3)$ & 30.9 \\
\hline Pions & 0.1 & $3.73 \times 10^{-1}(7.5)$ & $4.47 \times 10^{-1}(7.3)$ & 19.9 \\
\hline Neutrons & 57 & $6.1 \times 10^{-1}(12.3)$ & $7.1 \times 10^{-1}(11.6)$ & 16.3 \\
\hline Muons & 0.03 & $1.14 \times 10^{-1}(2.3)$ & $1.27 \times 10^{-1}(2.1)$ & 11.5 \\
\hline Total & $\mathbf{1 0 0}$ & $\mathbf{4 . 9 5}$ & $\mathbf{6 . 1 2}$ & $\mathbf{2 3 . 6}$ \\
\hline
\end{tabular}

that only a small and most probably not measurable influence of the RadFET cover is expected.

Detailed results for the liquid argon calorimeter are presented in Table III. The spectrum significantly differs from the one in the inner detector: it is composed almost only of photons and neutrons, and the absolute number of particles per unit area and time is around 70 times less than for the inner detector position. The annual TID expected in the liquid argon calorimeter is around (4.95 \pm 0.08$) \mathrm{Gy} /$ year without cover and $(6.12 \pm 0.09)$ Gy/year with cover: this corresponds to a dose enhancement of $(23.6 \pm 2.4) \%$ which can be interpreted as a not negligible increase of measured dose.

Finally, it should be mentioned that the TID values obtained here without cover are consistent with the values evaluated by the ATLAS Radiation Background Task Force [51], that states an annual dose of $7.5 \mathrm{kGy} /$ year and $4.8 \mathrm{~Gy} / \mathrm{year}$ for the TID respectively deposited in Silicon devices for the SCTB 1 and LAR Barrel position. The compatibility of the two sets of results is remarkable, considering the different simulation tools and configurations in the two studies.

\section{CONCLUSION}

A Geant4 application to simulate the impact of packaging on dose measurements of RadFET dosimeters to be used in the 
LHC experiments has been developed. The application was used to understand the dose enhancement due to the package for electrons, muons, photons, neutrons, pions, protons inside the complex LHC radiation fields.

Based on a detailed study on the validity of the physics models used for simulation, it was verified that the Geant4 application is an adequate and reliable tool to investigate the packaging impact in the complex LHC radiation fields. For two specific locations inside the ATLAS experiment the TID was calculated. It was demonstrated that the effect of a $260 \mu \mathrm{m}$ thick Alumina cover over the RadFET can alter the predicted measured dose up to about 25\% in some locations of the LHC experiments. This relative dose enhancement between covered and uncovered RadFET is similar than the absolute accuracy of RadFET dose measurements one can expect to achieve in the radiation fields of LHC experiments; however, it represents a significant shift in the measured value. The choice of RadFET packages is thus an important issue for measuring the TID in High Energy Physics experiments.

\section{ACKNOWLEDGMENT}

For fruitful discussions and clarifying comments we thank Ian Dawson from the ATLAS Radiation Background Task Force, the members of the INFN Genoa Geant4 group, and the irradiation teams at the PIF Facility of the PSI in Villigen and the Reactor Research Center at the JSI in Ljubljana.

\section{REFERENCES}

[1] I. Dawson and C. Buttar, "The radiation environment in the ATLAS inner detector," Nucl. Instr. \& Meth., vol. 453, pp. 461-467, 2000.

[2] M. Huhtinen, "Radiation environment simulations for the CMS detector," CERN CMS Tech. Note 95-198, 1995.

[3] A. G. Holmes-Siedle and L. Adams, Handbook of radiation effects, 2nd ed. New York: Oxford University Press, 2002.

[4] LHC Experiment Radiation Monitoring Working Group (RADMON) [Online]. Available: http://lhc-expt-radmon.web.cern.ch/lhc-exptradmon

[5] F. Ravotti et al., "SENSOR CATALOGUE-data compilation of solid-state sensors for radiation monitoring," CERN TS-Note-2005-002, 2005.

[6] F. Ravotti et al., "Radiation monitoring in hadron environment at CERN: From the IRRAD6 facility to the LHC experiments," IEEE Trans. Nucl. Sci., vol. 54, pp. 1170-1177, 2007.

[7] I. Mandic et al., "Online integrating radiation monitoring system for the ATLAS detector at the large hadron collider," IEEE Trans. Nucl. Sci., vol. 54, pp. 1143-1150, 2007.

[8] D. Wiednerand and G. Corti, "LHCB radiation monitors for detectors and detector electronics," LHCb Eng. Note, CERN EDMS 860046, 2007.

[9] F. Ravotti et al., Technical and Functional Specification of the TOTEM on-line Radiation Monitoring System, CERN EDMS 8749452008 [Online]. Available: https://edms.cern.ch/document/874945

[10] F. Ravotti et al., "Response of RadFET dosimeters to high fluences of fast neutrons," IEEE Trans. Nucl. Sci., vol. 52, pp. 959-965, 2005.

[11] A. B. Keating et al., "Modelling packaging effects on proton irradiation response on NMRC RadFETs. New GEANT4 simulations and Co-60 irradiations," in Proc. RADECS conf., Noordwijk, 2003, pp. 457-463.

[12] A. B. Rosenfeld et al., "MOSFET dosimeters: The role of encapsulation on dosimetric characteristic in mixed-gamma neutron and megavoltage x-ray field," IEEE Trans. Nucl. Sci., vol. 42, pp. 1870-1877, 1995.

[13] G. J. Brucker, S. Kronenberg, and F. Genter, "Effects of package geometry, materials, and die design on energy dependence of pMOS dosimeters," IEEE Trans. Nucl. Sci., vol. 42, pp. 33-40, 1995.

[14] S. Agostinelli et al., "GEANT4-A simulation toolkit," Nucl. Instr. Meth. A, vol. 506, pp. 250-303, 2004.

[15] J. Allison et al., "Geant4 developments and applications," IEEE Trans. Nucl. Sci., vol. 53, pp. 270-278, 2006.
[16] F. Ravotti, "Development and Characterization of Radiation Monitoring Sensors for the High Energy Physics Experiments of the Cern LHC Accelerator (Thesis), CERN-THESIS-2007-013," , Univ. Montpellier II, , France, 2006.

[17] W. Hajdas et al., "Radiation effects testing facilities in PSI during implementation of the Proscan project," in Proc. 2002 IEEE Rad. Effects Data Workshop, 2002, pp. 160-164.

[18] R. R. Jeraj et al., "Research reactor benchmarks," in Nucl. Sci. Eng., 2003, vol. 145 , pp. $145-152$.

[19] M. Glaser et al., "Geant4 simulation for LHC radiation monitoring," in Conf. Rec. 2006 IEEE Nucl. Sci. Symp., pp. N35-4.

[20] S. Chauvie et al., "Geant4 low energy electromagnetic physics," in Conf. Rec. 2004 IEEE Nuclear Science. Symposium, pp. N33-165.

[21] S. Chauvie et al., "Geant4 low energy electromagnetic physics," in Proc. Comp. in High Energy Phys., Beijing, China, 2001, pp. 337-340.

[22] S. Giani et al., Geant4 Simulation of Energy Losses of Slow Hadrons 1999, INFN Report INFN/AE-99/20.

[23] Stopping Powers and Ranges for Protons and Alpha Particles Intl. Commission on radiation units and measurements, 1993, ICRU Report 49.

[24] H. Burkhardt et al., "Geant4 standard electromagnetic package," in Proc. 2005 Conf. on Monte Carlo Method, Am. Nucl. Soc., 2005.

[25] J. Apostolakis et al., "Geant4 low energy electromagnetic models for electrons and photons," INFN/AE-99/18, 1999.

[26] S. T. Perkins et al., "Tables and graphs of electron-interaction crosssections from $10 \mathrm{eV}$ to $100 \mathrm{GeV}$ derived from the LLNL evaluated electron data library (EEDL)," LLNL UCRL-50400, vol. 31, 1997.

[27] D. Cullen et al., "EPDL97: The evaluated photon data library, 97 version," LLNL UCRL-50400, rev. 5, vol. 6, 1997.

[28] K. Amako et al., "Comparison of Geant4 electromagnetic physics models against the NIST reference data," IEEE Trans. Nucl. Sci., vol. 52, pp. 910-918, Aug. 2005.

[29] NIST Physical Reference Data [Online]. Available: http://physics.nist. gov/PhysRefData/

[30] A. Lechner, M. G. Pia, and M. Sudhakar, "Validation of Geant4 low energy electromagnetic processes against precision measurements of electron energy deposit," IEEE Trans. Nucl. Sci.,., 2009.

[31] H. H. Andersen and J. F. Ziegler, The Stopping and Ranges of Ions in Matter. Oxford: Pergamon Press, 1977.

[32] J. F. Ziegler, J. P. Biersack, and U. Littmark, The Stopping and Ranges of Ions in Solids. Oxford, U.K.: Pergamon Press, 1985.

[33] J. F. Ziegler, "SRIM-2003," Nucl. Instr. Meth. B, vol. 219-220, pp. 1027-1036, 2004.

[34] A. Lechner and M. G. Pia, "Analysis of Geant4 physics models for the simulation of proton depth dose profiles in radiotherapeutical applications," IEEE Trans. Nucl. Sci., 2008.

[35] A. G. Bogdanov et al., "Geant4 simulation of production and interaction of muons," IEEE Trans. Nucl. Sci., vol. 53, pp. 513-519, 2006.

[36] L. Urban, "A model for multiple scattering in Geant4," in Proc. of The Monte Carlo Method, Am. Nucl. Soc., La Grange Park, IL, 2005.

[37] H. S. Fesefeldt, "The simulation of hadronic showers: Physics and applications," PITHA-85-02, RWTH Aachen, Sep. 1985.

[38] G. Folger, V. N. Ivanchenko, and J. P. Wellisch, "The binary cascade," Eur. Phys. J. A., vol. 21, pp. 407-417, 2004.

[39] G. Folger and J. P. Wellisch, "String parton models in Geant4," in Proc. Comp. in High Energy and Nucl. Phys., La Jolla, CA, Mar. 2003.

[40] R. K. Tripathi et al., "Universal parameterization of absorption cross sections," in NASA Technical Paper 3621, Jan. 1997.

[41] R. K. Tripathi et al., "Accurate universal parameterization of absorption cross sections," Nucl. Instr. Meth. B, vol. 117, no. 4, pp. 347-349, 1996.

[42] R. K. Tripathi et al., "Universal parameterization of absorption cross sections-light systems," in NASA Technical Paper 1999-209726, 1999.

[43] R. K. Tripathi et al., "Universal parameterization of absorption cross Section III-light systems," Nucl. Instrum. Meth. B, vol. 155, no. 4, pp. 349-356, Sep. 1999.

[44] W. Q. Shen et al., "Total reaction cross section for heavy-ion collisions and its relation to the neutron excess degree of freedom," Nucl. Phys. A, vol. 491, no. 1, pp. 130-146, 1989.

[45] V. Ivanchenko et al., "The Geant4 hadronic verification suite for the cascade energy range," Proc. Comp. in High Energy Phys., 2003.

[46] J. P. Wellisch, "Geant4 hadronic physics status and validation for large HEP detectors," in Proc. Comp. High Energy Phys., La Jolla, CA, 2003.

[47] T. Ersmark et al., "Status of the desire project: Geant4 physics validation studies and first results from columbus/ISS radiation simulations," IEEE Trans. Nucl. Sci, vol. 51, pp. 1378-1384, 2004. 
[48] D. H. Wright et al., "Recent developments and validations in Geant4 hadronic physic," in AIP Conf. Proc., Oct. 2006, vol. 867, pp. 479-486.

[49] A. N. Kolmogorov, "On the empirical determination of a distribution law," Gior. Ist. Ital. Attuari, vol. 4, pp. 83-91, 1933.

[50] N. V. Smirnov, "On the estimation of the discrepancy between empirical curves of distributions for two independent samples," in Bull. Math. Univ., Moscow, 1939.

[51] Radiation Background Task Force. Results from GCALOR with the Baseline Shielding Layout Jun. 2003 [Online]. Available: http://atlas. web.cern.ch/

[52] T. Tabata and R. Ito, "A generalized empirical equation for the transmission coefficient of electrons," Nucl. Instr. Meth., vol. 127, p. 429, 1975.

[53] M. J. Berger, J. H. Hubbell, S. M. Seltzer, J. S. Coursey, and D. S. Zucker, XCOM: Photon Cross Section Database. Gaithersburg, MD: NIST, 1999.
[54] C. Leroy and P. G. Rancoita, Principles of Radiation Interaction in Matter and Detection. Singapore: World Scientific Publishing Co. Pte. Ltd, 2004.

[55] M. Kossov, "Chiral-invariant phase space model," Eur. Phys., vol. 14, no. 3, pp. 265-269, Jul. 2002.

[56] P. V. Degtyarenko et al., "Chiral invariant phase space event generator," Eur. Phys. J. A., vol. 9, no. 3, pp. 411-420, 2003.

[57] A. Ferrari, P. R. Sala, A. Fassó, and J. Ranft, Fluka: A Multi-Particle Transport Code Geneva, 2005, CERN-2005-010.

[58] A. F. Fassó et al., "The physics models of fluka: Status and recent developments," Proc. Comp. in High Energy Phys., 2003.

[59] C. Zeitnitz and T. A. Gabriel, "The GEANT-CALOR interface and benchmark calculations of ZEUS test calorimeters," Nucl. Instr. Meth. A, vol. 349, no. 1, pp. 106-111. 